\title{
Studies on Identification of the Food Born Fungal Pathogens and their Pathogenic Effects on Human Health
}

\author{
Nadia Jabeen ${ }^{1 *}$ and Khuram Shahzad ${ }^{2}$ \\ ${ }^{1}$ Department of Agriculture, Hazara University, Khyber Pakhtun Khawa (KPK), Pakistan \\ ${ }^{2}$ Department of Agronomy, Agricultural University Peshawer, Khyber Pakhtun Khawa (KPK), Pakistan \\ *Corresponding Author: Nadia Jabeen, Department of Agriculture, Hazara University, Khyber Pakhtun Khawa (KPK), Pakistan.
}

Received: September 12, 2019; Published: September 26, 2019

DOI: 10.31080/ASAG.2019.03.0667

\begin{abstract}
The present review research was conducted to find out the quality of food available at surrounding food spots in front gate of Hazara University. Samples were taken from different burger shops and few vegetables and dry fruits seller. The fugal pathogens were identified by culturing in the Microbiology lab and studied for characterization on morphological basis and from the literature [1,2]. It was observed that mostly shopkeepers were using expire dated bread, not showing the symptoms but having the pathogens. The vegetables were also contaminated with Aspergillus niger and Aspergillus flavus.

Keywords: Pathogen; Human Health; Aspergillus niger; Aspergillus flavus
\end{abstract}

\section{Introduction}

Fungi is included in the plant kingdom, they lack chlorophyll, chemo heterotrophs and lack of mobility. Fungal cell contains membrane bound cell organelles including nuclei, mitochondria, Golgi apparatus, endoplasmic reticulum and lysosomes and are eukaryotic. They exhibit mitosis. They have ergosterols in their membranes and possesses 80s ribosome. Fungi are osmotrophic they obtain their nutrients by absorption. They obtain nutrients as saprophytes (live off of decaying matter) or as parasites (live off of living matter). All fungi require water and oxygen and there are no obligate anaerobes. Fungi typically reproduce asexually and/ or sexually by producing spores. They grow either reproductively by budding or non-reproductively by hyphal tip elongation. Food storage is generally in the form of lipids and glycogen. Fungi lack chlorophyll therefore they do not carry out photosynthesis. Fungi obtain their food by secreting digestive enzymes onto the substrate and then absorbing the organic molecules that are released by the enzymes [3].

Fungi are basically filamentous in their growth form and their bodies consist of long slender filaments called hyphae. Most fungi reproduce sexually with nuclear exchange rather than gametes. The cell wall of fungi is built of polysaccharides (chains of sugars) and chitin. Mitosis in fungi is different from that in plants or most other eukaryotes. The nuclear envelope does not break down and re-form. Instead mitosis takes place within the nucleus.

The hyphae are made up of long chains of cells joined end-toend divided by cross-walls called septa (singular, septum). Fungal hyphae grow very rapidly when food and water are abundant and the temperature is optimum. A mass of connected hyphae is called a mycelium (plural, mycelia). Three kinds of reproductive structures occur in fungi: (1) Sporangia, which are involved in the formation of spores; (2) Gametangia, structures within which gametes form; and (3) Conidiophores, structures that produce conidia, multinucleate asexual spores. Spores are a common means of reproduction among fungi. They may form as a result of either asexual or sexual processes and are always non-motile, being dispersed by wind. When spores land in a suitable place, they germinate, giving rise to a new fungal hyphae.

\section{Food born fungi}

The thousands of genera and species of Fungi occurring in foods may be divided in bacteria, molds and yeast. Changes in foods are mediated by a variety of microbes that use food as a carbon and energy source. These organisms include prokaryotes (bacteria), single-celled organisms lacking defined nuclei and other organelles, and eukaryotes, single-celled (yeasts) and multicellular (molds) organisms with nuclei and other organelles and flavors.

Yeasts

Yeasts are a subset of a large group of organisms called fungi that also includes molds and mushrooms. Yeasts are the single celled colorless and bud forming. The cell shape is round or oval they are able to grow in the absence of air. The yeasts are found in food are divided into classes: Ascomycetes and fungi imperfect. They are responsible for spoilage of several foods e.g fruits may be caused by these organisms. The production of beer, wine and other alcoholic beverages is based on fermentative activity of yeasts.

There are four main groups of spoilage yeasts

- Zygo saccharomyces and related genera tolerate high sugar and high salt concentrations and are the usual spoilage organisms in foods such as honey, dried fruit, jams and soy 
sauce. They usually grow slowly, producing off-odors and flavors and carbon dioxide that may cause food containers to swell and burst.

- $\quad$ Saccharomyces spp is best known for their role in production of bread and wine. Some species grow on fruits, including yogurt containing fruit. Candida and related genera are a heterogeneous group of yeasts, some of which also cause human infections. They are involved in spoilage of fruits, some vegetables and dairy products.

Molds are filamentous fungi that do not produce large fruiting bodies like mushrooms. Molds are very important for recycling dead plant and animal remains in nature but also attack a wide variety of foods and other materials useful to humans. Molds are multicellular, filamentous fungi and its growth on foods is recognized by its fuzzy or cottony appearance. Molds generally causing spoilage of food and grow well at room temperatures usually between $25-30^{\circ} \mathrm{c}$. They require free oxygen for their growth due to which they grow on the surface of contaminated food. Zygomycetes are considered relatively primitive fungi but are widespread in nature. Generally they require high water activities for growth and are notorious for causing rots in a variety of stored fruits and vegetables, including strawberries and sweet potatoes. Some common bread molds also are zygomycetes. The most common spoilage species are Mucor and Rhizopus. Zygomycetes are not known for producing mycotoxins but there are some reports of toxic compounds produced by a few species. Rhizopus Nigerians sometimes called bread molds is a very common mold occurring in foods. It is involved in the spoilage of many foods such as berries, fruits, vegetables and bread etc.

Penicillium and related genera are present in soils and plant debris from both tropical and Antarctic conditions but is likely to dominate spoilage in temperate regions. They are distinguished by their reproductive structures that produce chains of conidia. Although they can be useful to humans in producing antibiotics and blue cheese, many species are important spoilage organisms, and some produce potent mycotoxins (patulin, ochratoxin, citreoviridin, penitrem). Penicillium spp. cause visible rots on citrus, pear, and apple fruits and cause enormous losses in these crops. They also spoil other fruits and vegetables.

Aspergillus and related molds generally grow faster and are more resistant to high temperatures and low water activity than Penicillium spp. and tend to dominate spoilage in warmer climates.

Aspergillus also involved in spoilage of food [4].

Many aspergilla produce mycotoxins: aflatoxins, ochratoxin, territrems and cyclopiazonic acid. Aspergilla spoil a wide variety of food and non-food items (paper, leather etc.) but are probably best known for spoilage of grains, dried beans, peanuts, tree nuts, and some spices.

Other molds, belonging to several genera, have been isolated from spoiled food. These generally are not major causes of spoilage but can be a problem for some foods [4].

\section{Toxins}

Fungal poisonous secondary Metabolites called mycotoxins. There are five mycotoxins, or groups of mycotoxins, that occur in food: deoxynivalenol/nivalenol; zearalenone; ochratoxin; fumonisins; and aflatoxins. Toxin-producing species of genera Aspergillus (A. carbonarius, A. flavus, A. ochraceus, A. oryzae, A. parasiticus, A. versicolor), Penicillium ( $P$. nordicum, $P$. expansum, $P$. viridicatum, $P$. verrucosum), Fusarium (F. culmorum, F. graminearum, F. oxysporum, F. verticillioides, F. proliferatum), Alternaria (A. alternata, A. solani, $A$. brassicae, A. tenuissima, A.tomato) as well as teleomorphs of the class Ascomycetes (Petromyces alliaceus, Emericella nidulans, etc.) can biosynthesize toxic secondary metabolites - mycotoxins (aflatoxins, ochratoxin A, sterigmatocystin, patulin, fumonisins, zearalenone, deoxynivalenol, alternariol, alternariol monomethyl ether, tenuazonic acid) [5].

\section{Mode of action}

The ingestion of such mycotoxin by animals and human beings has enormous public health significance because these toxins are nephrotoxic, immunotoxic, teratogenic, mutagenic, cytotoxicity, hepatotoxicity, neurotoxicity and cancerogenity which are capable of causing acute and chronic effects in man and animals ranging from death to disorder of central nervous, cardiovascular and pulmonary systems and intestinal tract. Of greatest concern is the relevance of these toxins in human hepatoma and oesophageal cancer, increased susceptibility to diseases especially in children and childhood pre-five mortality and reduced life expectancy. At a cellular level, mycotoxins react with nucleic acids and inhibit the biosynthesis of macromolecules DNA and RNA, or act on structures and functions of biological membranes or impair the energy metabolism (Dimić., et al. 2013).

\section{Food spoilage}

Food spoilage is a metabolic process that causes foods to be undesirable or unacceptable for human consumption due to changes in sensory characteristics. Spoiled foods may be safe to eat, i.e. they may not cause illness because there are no pathogens or toxins present but changes in texture, smell, taste, or appearance cause them to be rejected. Some spoilage is usual, and a variety of factors cause deterioration of foods. Endogenous enzymes in plants oxidizing phenolic compounds (browning) or degrading pectins. These factors are interrelated, as certain temperatures and oxygen and moisture levels increase the activities of endogenous enzymes and of microbes. Rodent and insect damage may provide an entry point for microbial growth.

Spoilage of food is identified by off-colors, off-odors, softening of vegetables, fruits and slime. However, even before it becomes obvious, microbes have begun the process of breaking down food molecules for their own metabolic needs. Sugars and easily digested carbohydrates are used first, plant pectins are degraded. Then proteins are attacked, producing volatile compounds with characteristic smells such as ammonia, amines and sulfides [4]. 


\section{Review of Literature}

Colin. V. L., et al. in 2013 conducted his worked on fungal morphology of $A$. niger and produces fungus by altering the hyphal morphology and the conidia adhesion capacity through biotechnological applications. They observed that Current problems of filamentous fungi fermentations and their further successful developments as microbial cell factories are dependent on control fungal morphology [1].

Gupta. M., et al. in 2012 carried out his worked on effect of various types on the rates of growth A. niger. They told that $A$. niger is a common soil borne fungus is industrially important and utilized in the fields of biotechnology and food microbiology. They compared the rate of growth of $A$. niger in various media types viz. simple PDA (Potato Dextrose Agar), modified PDA (containing additional sucrose and peptone), CYA (Czapek's Dox + Yeast Extract Agar), and LCA (Lignocellulose Agar). They concluded that PDA is the most commonly used laboratory medium for fungi due to its good and balanced nutrient content. However, when optimized or modified by nutrients viz. sucrose and peptone, it gives better results of fungal growth rate. The LCA medium is however secondary while CYA has been considered as average medium for fungal growth rate [6].

Sharma. R in 2012 carried out his worked on pathogenicity of $A$. niger in plants. A. niger is a member of the genus Aspergillus which is reproduce by asexual and sexually also. Aspergilli are everywhere in nature. A. niger is commonly found as a saprophyte growing on dead leaves, stored grain, compost piles and other decaying vegetation. He identified microscopically that its conidiophores are smooth-walled, hyaline or turning dark towards the vesicle and conidia are globose to subglobose (3.5-5.0 um in diameter), dark brown to black and rough-walled. And conidium is responsible for pathogenicity in various species of plants, which can be treated by antibiotics, chemicals and antibiosis. Biological control however is the best and most effective treatment [7].

Meijer. M., et al. in 2011 conducted his worked on growth and hydrolase profiles which can be used to distinguish $A$. niger and other black aspergilli. They compare wild type $A$. niger isolates from all over the world to each other and to the type strains of other black Aspergillus species with respect to growth and extracellular enzyme profiles. His result was only small differences were found in the extracellular enzyme profile of the A. niger isolates during growth on wheat bran, while large differences were observed in the profiles of the different black aspergilli. In addition, they also observed differences in temperature profiles between the black Aspergillus species. These data indicate that the local environment does not affect $A$. niger with respect to growth profile or enzyme production.

Silva. M.D., et al. in 2011 his work aimed is to distinguish Aspergillus species of the Nigri Section from foods and grains by utilizing morphologic and physiologic characters. The isolation of the species of the Nigri Section on Creatine Sucrose Agar (CREA) enabled to distinguish the Aspergillus sp species, which was characterized by the lack of sporulation and by the production of sclerotia [8].
Saori Amaike S., et al. in July 2009 conducted his work on distinct roles for VeA and LaeA in development and pathogenesis of A. flavus. Two proteins VeA and LaeA known to form a nuclear complex veA and laeA alleles discovered critical roles for VeA and LaeA in A. flavus development and seed colonization. This study concluded the unique roles of VeA and LaeA in seed pathogenesis and fungal biology [9].

Frisvad j.c., et al. in 2007 carried out his worked on Fumonisin B2 Production by A. niger. A. niger produced fumonisin B2 best on agar media with a low water activity. A. niger is used for the production of citric acid and as producer of extracellular enzymes, and also as a transformation host for the expression of heterologous proteins and they concluded that certain strains of $A$. niger produce both ochratoxin A and fumonisins so some foods and feeds may potentially contain two types of carcinogenic mycotoxins [10].

Hedayati. M.T., et al. in 2007 conducted his worked on A. flavus: human pathogen, allergen and mycotoxin producer. After conducted his experiment in mice he concluded that $A$. flavus is the second leading cause of invasive aspergillosis and it is the most common cause of superficial infection. Experimental invasive infections in mice show A. flavus to be 100-fold more virulent than A. fumigatus. A. flavus produces aflatoxins, the most toxic and potent hepatocarcinogenic natural compound. He also observed that accurate species identification remains difficult due to overlapping morphological and biochemical characteristics [11].

Mellon. J.E., et al. in 2007 carried out his worked on A. flavus hydrolases and their roles in pathogenesis and substrate utilization. A. flavus is a fungus saprophytic in mode, opportunistic pathogen with a wide non-specific host range (plants, animals, and insects) and produces carcinogen aflatoxin. For nutrient it penetrates in the host tissues. A. flavus produce extracellular hydrolases. Many hydrolases are presumed to function in polymer degradation and nutrient capture. Proteinases degrade protein substrates, such as elastin that is found in mammals and insects, but may also play roles in fungal defense and virulence. In some hosts, secretion of aamylase is critical for starch digestion and may play a critical role in induction of aflatoxin biosynthesis [12].

Hernández-Lauzardo.AN., et al. in 2005 the objectives of the study were to evaluate the growth rate and sporulation of three strains of $R$. stolonifer on different media and to measure the sporangiophores, sporangia and sporangiospores of three strains by image analysis, determining the relative frequencies of spore shapes, and by scanning electron microscopy, to confirm their shape and ornamentation. Results of this study indicated a better definition of mycelia, sporangia, and sporangiospores when isolates were grown on potato-dextrose-agar (PDA) [13].

Dai. Z., et al. in 2004 they carried out his worked on identification of genes associated with morphology in A. niger by using suppression subtractive hybridization. The morphology of citric acid production strains of $A$. niger is sensitive to a variety of factors, including the concentration of manganese (Mn). Upon increasing the $\mathrm{Mn} 2$ concentration in $A$. niger cultures to $14 \mathrm{ppb}$ or higher, due to 
which rapid decline in citric acid production. The molecular mechanisms through which Mn2 exerts effects on morphology and citric acid production in A. niger cultures have not been well defined, but our use of suppression subtractive hybridization has identified 22 genes responsive to Mn2. They used Northern blot analysis which showed that the transcripts of all 22 genes were rapidly enhanced or suppressed by Mn2 [14].

Schuste. E., et al. in 2002 carried out his worked on safety of A. niger. They told that it has been producing extracellular (food) enzymes, citric acid, biotransformation and in waste treatment and they also compared with other filamentous fungi and that it does not cause problem concerning allergy or mycopathology but cause a lung infections in severely immune-compromised patients. They observed that in tropical areas, ear infections (otomycosis) occur due to $A$. niger invasion of the outer ear canal but this may be caused by mechanical damage of the skin barrier. A. niger strains produce a series of secondary metabolites ochratoxin A. So he concluded that only $3-10 \%$ of the strains were examined for ochratoxin A production under favorable conditions and with these limitations A. niger is a safe production organism [15]

Lorbeer J.W., et al. in 2002 They carried out his worked on the nature and source of inoculum of A. niger causing the Aspergillus Black Mold disease of onion seed stalks and flower parts and they concluded from the results that when onion seed is infested by $A$. niger solely on the surface of the seed a fungicide seed treatment might be used to control future growth of the fungus. And by using this method A. niger was present on only a low percentage of the seeds [16].

Kwon. J.H., et al. in 2001 carried out his work on Rhizopus soft rot on cherry tomato caused by R. stolonifer in Korea and from the result they identified its pathogenesis and colonies [17].

Omoifo. C.O in 2011 carried out his worked on dimorphism in $R$. stolonifer. The conclusion from this study is that $R$. stolonifer could be polymorphic [2].

Van etten. J.L., et al. in 1973 they carried out his worked on physiological and morphological correlation of $R$. stolonifer spore germination. Sporangiospores of $R$. stolonifer were examined at various stages of germination by scanning electron and phasecontrast microscopy. And the result of these observations was correlated with changes in spore dry weight, spore volume, respiration, and syntheses of ribonucleic acid, deoxyribonucleic acid, and protein during germination.

\section{Results}

Few foodborne fungal pathogens were identified from food samples and review writing was done.

\section{Identified species}

Aspergillus niger

The member of the genus Aspergillus is Aspergillus niger which includes a set of fungi that are generally considered asexual, although perfect forms (forms that reproduce sexually) have been found [7]. Stipes color is slighty brown, surface is smooth, vesicle serration is large size, shape is glubose and conidia surface is very rough irregular [18]. It is known to create increased amount of pathogenicity in various species. A. niger is a fungus and one of the most common species of the genus Aspergillus. It causes a disease called black mold on certain fruits and vegetables such as grapes, onions and peanuts and is a common contaminant of food. Due to sophisticated nature, human beings gets frequently exposed to spores and vegetative forms of $A$. niger present in air, on foodstuffs and others stored consumables products and suffers with allergic problems. A. niger may also produce certain mycotoxins which are heptocarcinogenic, nephrogenic immunological in nature. It is an important step to understand the diversity, pathogenicity and toxicology of this important spoilage A. niger. A. niger (black mold), a filamentous ascomycete having ability of fast growth and $\mathrm{pH}$ tolerance is most important cosmopolitan fungi associated with postharvest decay of different substrates. A. niger has been found to be opportunistic reason for infections of humans. A. niger also causes the rotting of numerous fruits, vegetables and other food products, thus causing substantial economic losses due to spoilage. For example, black rot of onions associated with A. niger is responsible for serious losses of onion bulbs in the field and in storage [7].

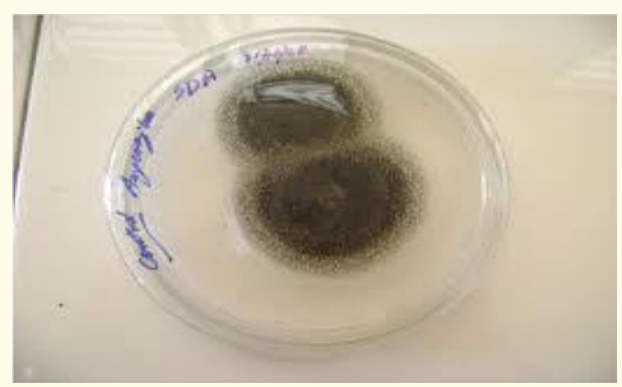

Figure 1: Aspergillus niger.

\section{Pathogenicity and affect on human health}

A. niger is generally regarded as a non-pathogenic fungus widely distributed in nature. Humans are exposed to its spores every day without disease becoming apparent. Only in few cases has A. niger been able to colonise the human body as an opportunistic invader and in almost all these cases the patients have a history of severe illness or immunosuppressive treatment. Several experimental studies to demonstrate the pathogenic potential of $A$. niger have come to the conclusion that neither ingestion of large doses of spores nor inhalation of spores induces mycosis in experimental animals. One day after ingestion, $A$. niger was no longer detected in the digestive tract, although ingested A. nidulans was isolated from the intestine of the animals. In contrast to A. fumigatus, this is known to be pathogenic A. niger showed no significant effect on the animals in the inhalation study. Compromising the immune system by steroid hormones seems to promote the spreading of the fungus in the body after an infection. Conducted a study with mice infected intravenously with high doses of $A$. niger isolated from sputum. They found evidence of pathogenic action only in groups that had been treated with a hydrocortisone drug. Addition of Decadron, a steroid hormone, to the culture medium of $A$. niger induced more 
vigorous corneal ulceration in rabbit eyes infected with spores compared to animals inoculated with spores from medium without the steroid. The authors conclude that exposure of the ordinarily harmless fungus to the steroid made them behave like a pathogen [15].

\section{Rhizopus stolonifer}

Rhizopus stolonifer commonly causes postharvest diseases on many fruits and vegetables. After comparing the growth rate and sporulation of three strains of R. stolonifer on different media and to quantify the sporangiophores, sporangia, and sporangiospores of three strains by image analysis, determining the relative frequencies of spore shapes, and by scanning electron microscopy, to confirm their shape and ornamentation. When mycelia, sporangia and sporangiospores were grown on potato-dextrose-agar (PDA) the growth rate and sporulation was higher on this culture medium. Values of length, diameter, and area of sporangiophores, sporangia, and sporangiospores varied among isolates [19].
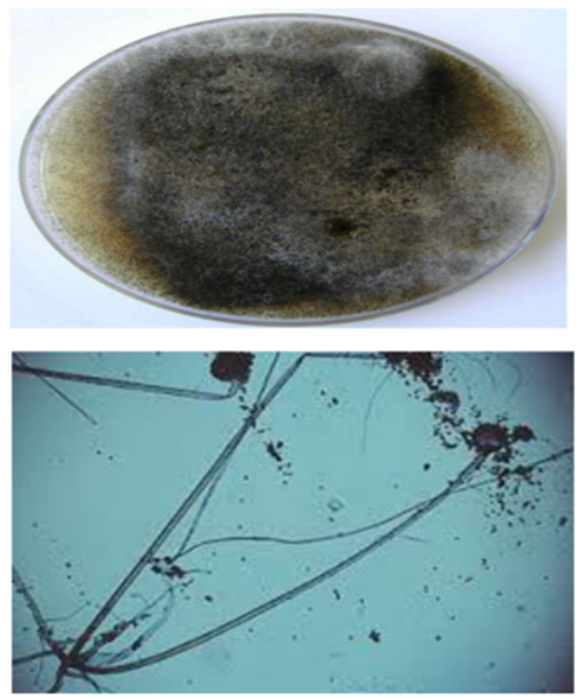

Figure 2: Rhizopus stolonifera.

When the mold comes in contact with a substrate, such as bread, it Fust spreads over the surface and penetrates it while sending hyphae inward to absorb the nutrients. $R$. stolonifer grows primarily as mycelia, which consists of long filamentous cells, or hyphae, that lack cross walls, known as septa. The lack of septa enables the mold to be named coenocytic. Coenocytic means that the mold is a multinucleate cell enclosed by one cell wall that contains chitin. R. stolonifer spores are usually dispersed in hot dry weather and they contain allergic proteins, which can cause respiratory and nasal symptoms in humans, such as coughing, chest discomfort and allergic reactions [20].

\section{Pathogenicity and effect on human health}

The fungal pathogen $R$. stolonifer causes a disease in food storage. The pathogens were isolated, identified and cultures were used to confirm their pathogenicity in their respective host. Exposure on consumption of this spoiled food may be responsible for serious health hazards.

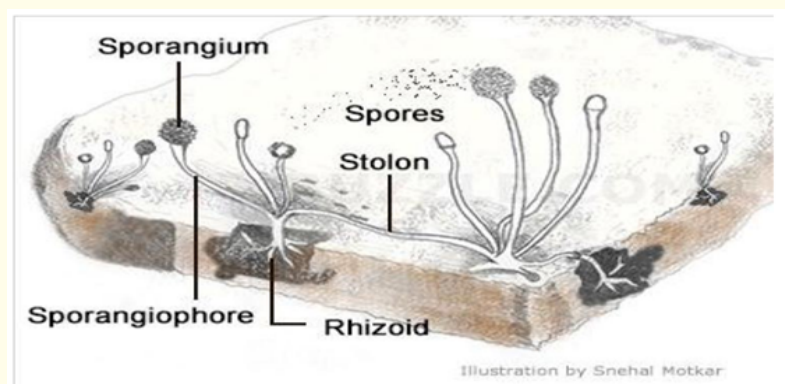

Figure a

Which may causes hazardous effects on human health [21]. When the spores of R. stolonifer land on a piece of bread or fruit, they will grow into full bodies of fungus. This spore could be carried through the air, picked up off of a hand or counter, or it could have come with the bread. Once on the bread, adjacent fungi will grow horizontal hyphae toward each other, and opposite mating types will sexually reproduce by forming gametes, and then they will combine to form a zygote. From the zygote a sporangium will grow and release spores to be picked up by another food source (biology 11, chapter 9).

\section{Aspergillus flavus}

Aspergillus flavus belongs to the Genus Aspergillus, Subdivision Deutoromycotina. A. flavus strains can cause disease in both plants and animals. Many fungi moved from opportunistic forms to specialized pathogens by gaining the ability to produce host-selective toxins. The hyphae are well developed, branch, septate, and hyaline; their cells are, multinucleate. The mycelium produces an abundance of conidiophores. The conidiophores are long, erect hyphae, each terminating in a bulbous head, the vesicle. Conidiophore of $A$. flavus with conidium-bearing cell. Conidia are the phialides reach maturity, they begin to form conidia at the tips, one below the other, in chains. The conidia of Aspergillus are formed inside of the phialide, which is actually a tube. A portion of the protoplasm with a nucleous at the tip of the sterigma is delimited by a septum. The conidia are unicellular with extremely roughened walls. A. flavus produces a variety of toxins including aflatoxins. Aflatoxins are harmful and are considered carcinogenic acute toxicity of aflatoxins in Homo sapiens has not been observed very often.

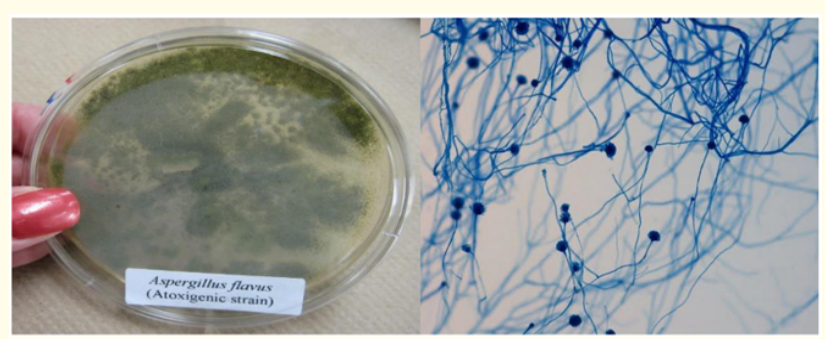

Figure 3: Aspergillus flavus. 
A. flavus causes aspergillosis, a life threatening human disease, particularly in patients who are immunosuppressed or have chronic lung disease. A. flavus is responsible for about $30 \%$ of the cases of aspergillosis.

\section{Pathogenesis and effect on human health}

Susceptibility to aflatoxicosis may be influenced by age, sex, nutritional status, health, and the level and duration of exposure. Humans can become sick by consuming unsafe levels of aflatoxin contaminated food and food products from grains, nuts and milk. Studies reveal that exposure to large doses (less than $6000 \mathrm{mg}$ ) of aflatoxin may cause acute toxicity. The target organ for aflatoxins is the liver. After the invasion of aflatoxins into the liver hepatocytes and leads to necrosis or liver cell death and decrease in liver function, there is a derangement of the blood clotting mechanism, jaundice, and a decrease in essential serum proteins synthesized by the liver. In animal experiments, AFB1 has been shown reduced Tlymphocyte function and number, suppress phagocytic activity, and reduce complement activity. Exposure to aflatoxin in contaminated food results in suppression of the cell-mediated immune response. Macrophages also increase their phagocytic activity and release various active products, such as cytokines and reactive intermediates, to carry out nonspecific immune responses. Several reports suggest that aflatoxin impairs the function of macrophages in animal species. Macrophages play a major role in host defenses against infection. Macrophages also increase their phagocytic activity and release various active products, such as cytokines and reactive intermediates, to carry out nonspecific immune responses [22-25].

\section{Conclusion}

It was concluded from the research review that the isolated species of fungi from the food items, badly affect the human heath by producing deteriorating components.

\section{Bibliography}

1. Colin VL., et al. "Tailoring fungal morphology of Aspergillus Niger MYA 135 by altering the hyphal morphology and the conidia adhesion capacity: biotechnological applications" (2013).

2. Omoifo CO. "Rhizopus stolonifer exhibits dimorphism". African Journal of Biotechnology 10 (2011): 4269-4275.

3. PN Rao. "Introduction to mycology" (2006).

4. Doyle ME. "Microbial Food Spoilage - Losses and Control Strategies" (2007)

5. Makun HA., et al. "Fungal and aflatoxin contamination of some" (2010).

6. Gupta M., et al. "Effect of various media types on the rate of growth of aspergillus niger". 2 (2012): 141-144.

7. Sharma R. "Pathogenecity of aspergillus niger in plants". 11 (2012): 47-51.

8. Silva DM., et al. "Identification of fungi of the genus Aspergillus section Nigria using Polyphasic Taxonomy". Brazilian Journal of Microbiology 42 (2011): 761-773.

9. Amaike S., et al. "Distinct Roles for VeA and LaeA in Development and Pathogenesis of Aspergillus flavus". 8 (2009): 10511060.

10. Frisvad JC., et al. "Fumonisin B2 Production by Aspergillus niger". Journal of Agricultural and Food Chemistry 55 (2007): 9727-9732.

11. Hedayati MT., et al. "Aspergillus flavus: human pathogen, allergen and mycotoxin producer". Microbiology 153 (2007): 1677 1692.

12. Mellon JE., et al. "Aspergillus flavus hydrolases: their roles in pathogenesis and substrate utilization". 77 (2007): 497-504

13. Hernández-Lauzardo AN., et al. "Identification of Rhizopus stolonifera (Ehrenb.: Fr.) Vuill., Causal Agent of Rhizopus Rot Disease of Fruits and Vegetables" (2005)

14. Dai Z., et al. "Identification of Genes Associated with Morphology in Aspergillus niger by Using Suppression Subtractive Hybridization". Applied and Environmental Microbiology 70 (2004): 2474-2485.

15. Schuster. "On the safety of Aspergillus niger". Applied Microbiology and Biotechnology 59 (2002): 426-435

16. Lorbeer JW., et al. "Nature and Source of inoculum of Aspergillus nigercausing the Aspergillus Black Mold Disease of Onions in New York" (2000).

17. Kwon JH., et al. "Rhizopus Soft Rot on Cherry Tomato Caused by Rhizopus stolonifer in Korea". 29 (3): 176-178.

18. Diba K., et al. "Identification of Aspergilus species using morphological characteristics". 23 (2007): 867-872.

19. Ana Niurka H., et al. "Identification of Rhizopus stolonifer: Causal Agent of Rhizopus Rot Disease of Fruits and Vegetables" (2005)

20. Olbrantz C. “Black Bread Mold Rhizopus stolonifer” (2011).

21. Bhale UN. "Survey of market storage diseases of some important fruits of Osmannabad District (M. S.) India". (2011): 88-91.

22. Anonymous. "Final risk Assessment for Aspergillus niger" (1997).

23. http://ci.vbi.vt.edu/pathinfo/pathogens/A-f.html

24. Kocić-Tanackov SD., et al. "Antifungal activity of essential oils in the control of food-borne fungi growth and mycotoxin biosynthesis in food". (2013): 838-849.

25. Meera G., et al. "Effect of various media types on the rate of growth of Aspergillus Niger". 2 (2012): 2231-6345.

Volume 3 Issue 10 October 2019

(C) All rights are reserved by Nadia Jabeen and Khuram Shahzad. 